\title{
The TESIS project Are type 2 QSO hidden in X-ray emitting EROs?
}

\author{
P. Severgnini ${ }^{1}$, R. Della Ceca ${ }^{1}$, V. Braito ${ }^{1}$, P. Saracco ${ }^{1}$, M. Longhetti ${ }^{1}$, R. \\ Bender $^{2}$, N. Drory ${ }^{3}$, G. Feulner ${ }^{2}$, U. Hopp ${ }^{2}$, F. Mannucci ${ }^{4}$, and C. Maraston ${ }^{5}$ \\ ${ }^{1}$ INAF-Osservatorio Astronomico di Brera (OAB), via Brera 28, 20121 Milano, Italy \\ ${ }^{2}$ Universitäts-Sternwarte München, Scheiner Str. 1, 81679 M-unchen, Germany \\ 3 University of Texas at Austin, Austin, Texas 78712 \\ ${ }^{4}$ IRA-CNR, Largo E. Fermi 5, 50125 Firenze, Italy \\ ${ }^{5}$ Max-Plank-Institut für Extraterrestrische Physik, Garching bei Munchen, Germany
}

\section{Introduction}

X-ray selected EROs are, on average, the hardest X-ray sources in medium and deep X-ray fields. This coupled with their extremely red colors $(\mathrm{R}-\mathrm{K}>5)$ suggest that they represent one of the most promising population where looking for high-luminosity $\left(\mathrm{L}_{X}>10^{44} \mathrm{erg} \mathrm{s}^{-1}\right)$ and X-ray obscured $\left(\mathrm{N}_{H}>10^{22} \mathrm{~cm}^{-2}\right)$ type2 AGNs, the so called QSO2 (e.g. [5]; [4]; Mignoli et al. submitted to A\&A). These latter are predicted in large density by the synthesis model of the Cosmic X-ray background ([3]) even if only few observational evidences have been found so far (e.g. [1] and references therein; Caccianiga et al. A\&A accepted).

With the aim at studying the properties of X-ray emitting EROs our group has recently gained $75 \mathrm{ksec}$ (AO-2 period) and $78 \mathrm{ksec}(\mathrm{AO}-3$ period) of XMMNewton observations centered on two MUNICS (MUNICS Near-IR Cluster Survey, [2]) fields of about $180 \mathrm{arcmin}^{2}$ each. For these fields photometric observations in the B, V, R, I, J, K' bands are available (Drory et al. 2001) down to limiting magnitudes of $\mathrm{R} \sim 24.5 \mathrm{mag}$ and $\mathrm{K} \sim 19.5 \mathrm{mag}$. All the EROs present in these 2 fields having a $\mathrm{K}^{\prime}$ magnitude brighter than 18.5 are already under investigation by the TESIS (TNG EROs Spectroscopic Identification Survey, Saracco et al. this conference and references therein) project.

Here we present the analysis of the XMM-Newton data for the S2F1 field and we discuss the X-ray properties of the 6 X-ray emitting EROs detected down to $\sim 10^{-15} \mathrm{erg} \mathrm{cm}^{2} \mathrm{~s}^{-1}$. We assume $\mathrm{H}_{0}=65 \mathrm{~km} \mathrm{~s}^{-1} \mathrm{Mpc}^{-1}$ and $\mathrm{q}_{0}=0$.

\section{X-ray data analysis}

The field S2F1 was observed by XMM-Newton on February 11, 2003. The data have been cleaned and processed using the Science Analysis System version 5.3.3. The net exposure time after data cleaning is about $41 \mathrm{ksec}$. We have cross-correlated the astrometrically corrected X-ray source catalogue with the TESIS-EROs catalogue. We found 5 secure X-ray emitting EROs and one more X-ray source which is more likely associated with an ERO counterpart. The detailed analysis of the 6 sources is presented in Severgnini et al. (in preparation). 
On the basis of their photometric redshifts all of the 6 X-ray emitting EROs have observed 2-10 keV luminosities $\left(\mathrm{L}_{(2-10 \mathrm{keV})}>10^{42} \mathrm{erg} \mathrm{s}^{-1}\right)$ and X-ray-tooptical flux ratios $\left(\log \left(\mathrm{F}_{(2-10 \mathrm{keV})}\right) / \log \left(\mathrm{F}_{R}\right)>-1\right)$ suggesting the presence of AGN. For three out of the 6 EROs we have been able to perform a complete X-ray spectral analysis. The X-ray data are well fitted by a single power-law model (Fig. 1).

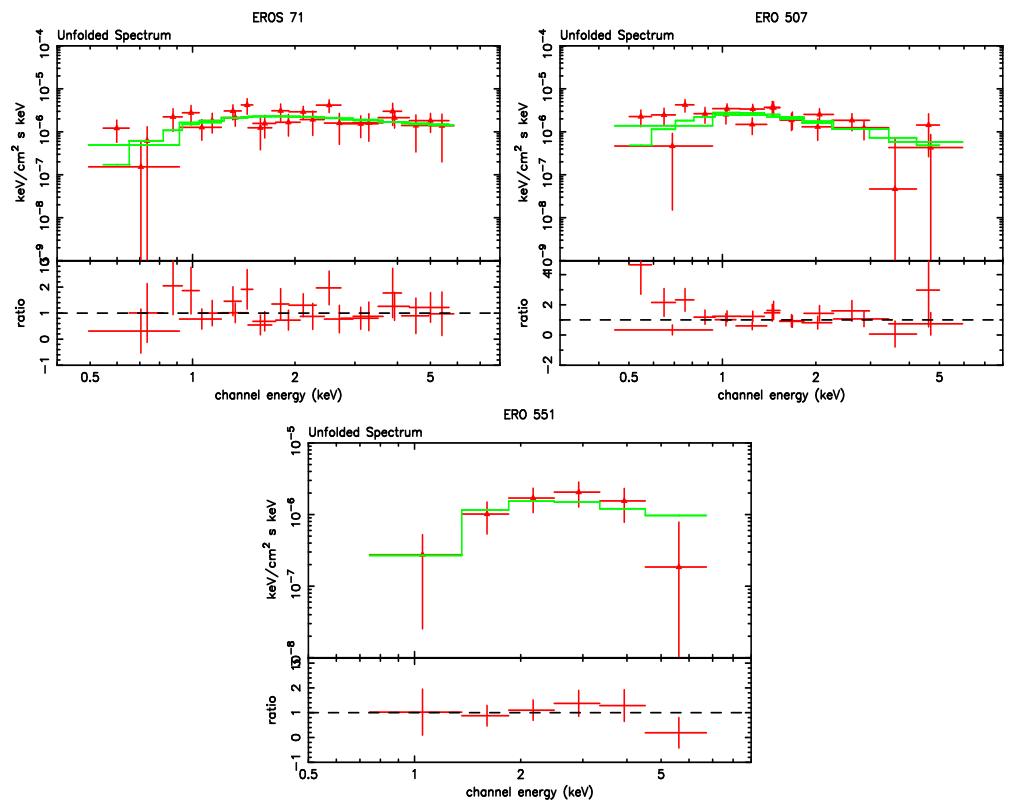

Fig. 1. XMM-Newton spectra in energy units (solid points) and best-fit model (continuous line). Ratios between data and the best-fit model values as a function of energy are reported in the lower panels.

A pure thermal component is rejected at more than $97 \%$ confidence level and the addition of a thermal component to the power-law model is not statistically required. These results, combined with the best fit parameters obtained, clearly indicate the presence of high luminosity (unabsorbed $\mathrm{L}_{(2-10 \mathrm{keV})}>10^{44} \mathrm{erg} \mathrm{s}^{-1}$ ), obscured $\left(\mathrm{N}_{H}>10^{22} \mathrm{~cm}^{-2}\right)$ AGN, i.e. QSO2 candidates.

\section{References}

[1] Della Ceca et al. 2003, A\&A 406, 555

[2] Drory et al. 2001, MNRAS 325, 550

[3] Gilli et al. 2001, A\&A 366, 407

[4] Stevens et al. 2003, MNRAS 342,

[5] Vignali et al. 2002, astro-ph/0209415 